\title{
EVA London 2020 Workshops
}

\author{
Nick Lambert \\ Ravensbourne University London \\ London, UK \\ n.lambert@rave.ac.uk
}

\author{
Carl H. Smith \\ Ravensbourne University London \\ London, UK \\ c.smith@rave.ac.uk
}

\begin{abstract}
This paper collects the various workshops that are currently planned for EVA London 2020. EVA London regularly features workshops, which provide a more 'hands-on' opportunity for delegates to learn new skills, from the EVA community. This year, Nick Lambert and Carl H. Smith have curated the workshops. Though the plans for these workshops are somewhat speculative due to the pandemic and postponement of the conference, yet we hope they will be included in some form, providing a valuable and enriching part of EVA London. This paper provides a documentation of the themes the workshops will address.
\end{abstract}

Glitch. Posthumanism. Machine learning. Virtual reality. Artificial imagination. Artistic intention. Facial expression.

\section{NEURAL BRAIN GLITCHES}

\section{Eyal Gruss, Israel}

Eyal Gruss is part of the tech-art group Mahanaim134, based in Tel Aviv. His work focuses on $\mathrm{Al}$ and generative art. He is currently working on new pedagogical creative tools to enable musicians, VJs and new media artists to access deep learning generative methods for manipulating visual imagery.

The workshop will consist of two 90-minute sessions long workshop, where we will introduce tools and methods to enable musicians, VJs and new media artists to access deep learning generative methods for manipulating visual imagery. The "glitch canvas" is a new pedagogical, creative and performative tool that can be activated with a midi controller, allowing the user the traverse and manipulate the latent space of generated faces and facial expressions in a semantic and informed way, without need of programming and machinelearning know-how.

The workshops will use open-source tools, best practices and Gruss's own approaches for solving inverse rendering and using pretrained generative models to create novel content.

Recent state-of-the-art generative algorithms such as BigGAN and SyleGAN are capable of generating diverse images of high-fidelity. However, these models are decoder - they generate images based on a latent list of numbers. They lack corresponding encoder - i.e. there is no structured way to find the numbers that would generate a desired image. Trying to find these numbers is known as the inverse rendering problem. This is a cheap alternative to retraining these models which is a costly and timely and data hungry procedure. Moreover, ability to semantically manipulate the latent space allows the creating of new output, including interpolation between images and semantic manipulation of certain attributes (e.g. smile, age, gender). Recent tools such as ArtBreeder allow creating interpolations and videos thereof using a simple web interface. However more complex reconstruction and manipulations require custom code.

Gruss will present his own techniques and his work WanderGAN that uses a real-time evolutionary search to guide BigGAN to re-imagine a photograph of a memory programming. 


\section{THE MILLENNIALS TOOLKIT}

\section{Carmel Barnea Brezner Jonas, Gabriel $S$ Moses \& Tsila Hassine} Israel and Germany

Desperately Seeking is a grassroots online-based community of concerned Millennials, formed in 2016 around themes relating to online mental and emotional health. Among its activities, the community offers workshops, online and offline pairing-sessions, personal digital services and symposia on the subject of 'associative seeking'. The community pursues an open-ended discussion on the fundamental question underpinning the human condition in the early 21st century: can the centralised services with which we navigate through the internet actually bring us happiness or can they only direct us to the closest search result?

DS workshop: why do you search? Do you do it in order to find out? To discover and explore? Or maybe the opposite, do you do it pre-emptively, driven by the fear of not being ready for what is out there? Does technology help you find answers to what really matters or does it just narrows down your range? By helping direct you to the quickest solutions, doesn't it also distract you from more creative ones? Sounds trivial? Maybe. Or maybe you've simply trivialised the most meaningful true goals, which underlie your pursuit?

The panel will focus on Desperately Seeking's personalised and group-based on and offline strategies for a balanced virtual diet. When digital detox fails, you can only opt for a more responsible way of feeding your addiction, choosing qualitative exploration over the quantitative dead-end search.

Shmoogle is a collective of artists who launched a new and subversive "rank-free" search engine. It eliminates the hierarchy imposed by engines such as Google and Bing, favouring random results.

\section{TECHNO HYPER-HUMANISM}

\section{Carl Smith \& Eddie Castañeda Ravensbourne University London}

The focus of this workshop is a new form of Hyperhumanism called Techno-Hyperhumanism which follows the current trend of 'Tech for Good' (of which many initiatives are springing up around the world including the 'Center for Humane Technology').
What is the difference between: Humanism, Transhumanism and Hyperhumanism (Posthumanism)?

(i) Humanism emphasises the value and agency of human beings, individually and collectively.

(ii) Transhumanism involves enhancement of all kinds (but humans are still considered to be the most important species).

(iii) Hyperhumanism (Posthumanism) involves the deconstruction of the human and understanding that the human condition is not a fixed, but an open notion.

As humans, we have become victims of our own technological advancement because we have used technology for convenience and comfort rather than self and societal improvement.

With the neurobiological decay caused by substituting our own cognition we have become a society that relies heavily on technology to accomplish actions that we used to do naturally.

Digital societies are entrapped by a technological feed that creates an enduring hyperreality for them. By breaking our perceptual and neurological patterns, we can open new neural pathways that allow us to understand the world in a more profound way.

This workshop will cover a variety of techniques on how to observe and understand the phenomena that makes up the reality we manage to perceive.

By carrying out different psychological and binaural neuro-hacking experiments, we will understand how our brain is able to accomplish apparently difficult tasks with great ease. The Neuro-hacking techniques include: Neurofeedback, Cognitive load reduction, Brain stimulation, Peripheral vision, and Awareness, Attention and Sensing exercises.

Questions to be addressed:

(i) How can we define what a Hyperhuman actually is?

(ii) What are some examples of Transhumanist tech and Techno-Hyperhumanist tech and what are the differences between them?

(iii) The quest for Hyperhumanity is not new but can we set a global standard in order to understand Hyperhumanity in a scientific, quantitative way?

(iv) How can we measure the amount of post humanity we need to survive on earth? 


\section{USING MACHINE LEARNING TO DESIGN MOVEMENT INTERACTION IN VIRTUAL REALITY}

\author{
Nicola Plant, Marco Gillies, Clarice \\ Hilton \& Michael Zbyszyński \\ Goldsmiths, University of London \\ Rebecca Fiebrink \& Phoenix Perry \\ University of the Arts London \\ Ruth Gibson \\ Coventry University \\ Carlos Gonzalez Diaz \\ University of York \\ Bruno Martelli \\ Studio Gibson/Martelli
}

\subsection{Introduction}

As immersive and interactive media is increasingly being adopted by artists, dancers and performers to create interactive installations and performance work, there is a demand for easy-to-use tools and ideation approaches that enables the design of movement interaction for immersive environments. We present ongoing work on an immersive tool immersive tool that uses interactive machine learning to recognise and implement complex movement interaction designs that are otherwise difficult to represent with programmed rules. We also explore techniques to equip users with embodied ideation design strategies to enable them to design compelling movement interfaces with the tool that hold the potential to engage full body interaction within immersive media.

\subsection{Immersive interaction design}

While movements such as picking up and interacting with virtual objects are straightforward to design, there are many forms of movement interaction that are not well supported by current technologies. Primarily those that cannot easily be defined mathematically and rely on recognising and interpreting embodied movements that are more performative or expressive. Machine learning is a promising approach to implementing more performative movement interaction styles because it can capture the complexity of these movements that are hard to represent in programmed rules. In addition, it does not require artists or performers to have a background in computer programming because it allows artists and performers to design and implement movement interaction using their bodies removing the barrier of programming.

This work presents a project developing a new immersive tool that uses interactive machine learning to recognise and implement complex movement interaction designs (Diaz et al. 2019).
The research aims to support independent developers and artists in designing movement and body-based interaction for Virtual Reality and immersive media, by building tools that allow designing by moving via Interactive Machine Learning.

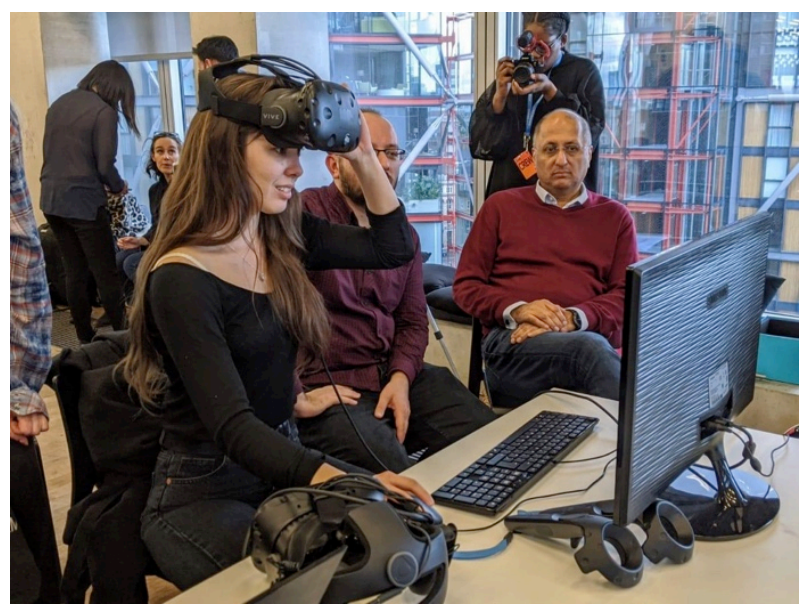

Figure 1: This image shows a workshop participant working with InteractML in Unity to train a machine learning model with a movement she performs.

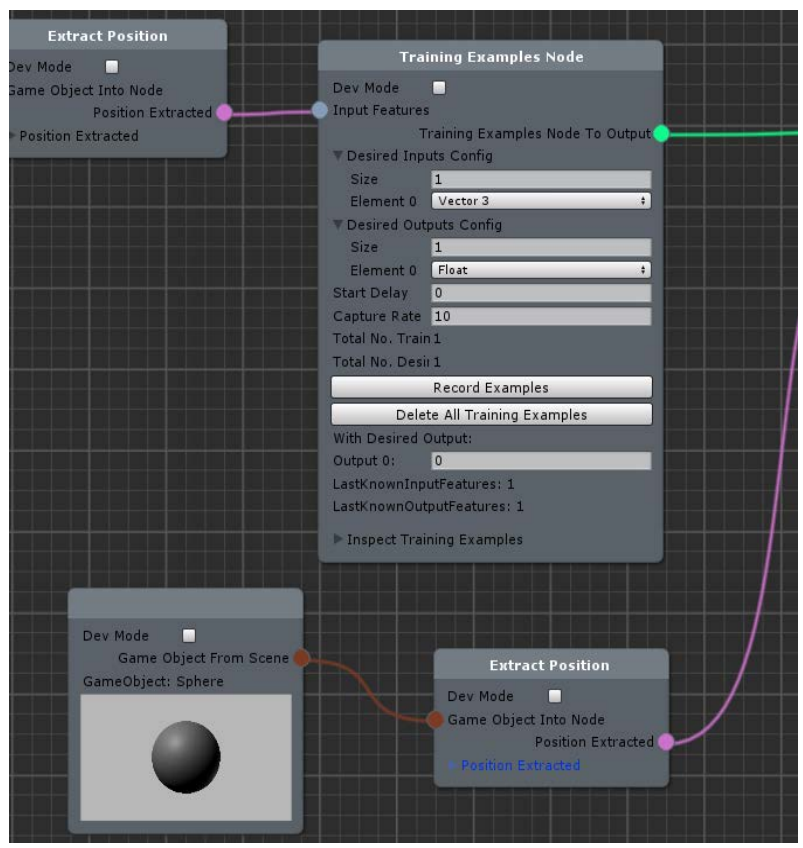

Figure 2: This image shows a section of the node-based graph that allows users to configure and train machine learning models within the tool.

\subsection{Workshop series}

User-centred design workshops supporting the project will be based on two techniques, one for design ideation and the second for implementation. The first is embodied sketching: designing movement interaction through movement (Segura et al. 2016). Rather than designing on paper, we will use our body movements to explore new ways 
interactivity in immersive environments. Developing movement interaction designs through physical "bodystorming" and "sketched" with our bodies, by acting them out (Höök 2018). If we are to carry on this embodied approach to design through to the implementation phase, we cannot just sit at a computer and start coding, we need to continue to use our body.

That is why we need our second technique, Interactive Machine Learning (Gillies 2019). Interactive Machine Learning uses machine learning technologies within an iterative design process that allows for rapid prototyping and refinement. This makes it possible for workshop participants to design and implement movement interaction by performing body movements.

The workshop will use the tool InteractML (interactml.com), developed to support interactive machine learning and movement interaction design in Unity3D (Diaz et al. 2019). By being able to configure and train parameters and models within a graph system, the tool requires no text-based programming experience to use it.

\subsection{Outcomes}

By creating better tools and working processes developers to enable those developers to create better movement interaction for players, audiences and end users. The tools will be tested and refined in the context of in-the-wild research with Immersive media creators. The aim of this research is to understand how interactive machine learning is used in real immersive creative work.

\section{WHENCE MUNCH'S SCREAM? TOWARD A MICROSCOPY OF ARTISTIC INTENTION}

\section{Derek J. Smith \\ Cardiff Metropolitan University (Ret'd)}

\subsection{Introduction}

At EVA 2014 (paper) and EVA 2016 (workshop), the author demonstrated how the Konrad artificial consciousness system (Smith 2014, 2016) could simulate the internals of visual-aesthetic cognition. This workshop offers delegates the opportunity to deploy the latest version of the Toronto-resident Konrad software in an exciting new way, namely to inform end-users as to the particular state of an artist's mind, in circumstances where that mind is no longer available to be interrogated directly.

\subsection{Session A - single-stimulus trials}

The workshop begins with a brief introduction to the cognitive neuroscience of facial expression. The received theory here is Ekman, Friesen, and
Hager's (2002) Facial Action Coding System, which allows for some 3000 discrete expressions, compiled by the activation of subsets of the 40-plus muscles of the face. Hypothetical control structures called "Action Units" coordinate the contraction of the different subsets. In humans, this processing is carried out subconsciously by deep brain structures; in the Konrad mainframe, it is simulated by four of the 21 modules of that system's processing hierarchy.

Konrad's simplest mode of operation is to respond from a resting state to end-user input specifying Action Unit (AU) and contraction intensity. A specific expression can be requested by selecting from a repertoire of the 50 commonest descriptors of emotional state in the English language. Muscle contraction intensities may be directly specified using a 10-point intensity scale from 0 to 9 . Delegates are invited to run individual 10-minute familiarisation jobs in this mode. Output to the enduser device is firstly posted on a cadaveric face to display the muscles required to produce the corresponding expression, and then used to construct a flesh-rendered final image. For example, a user who specified AU12 L2R1 would get the slightly asymmetrical mouth-only smile of Leonardo da Vinci's "Mona Lisa", as shown in Figure 3a. By invoking AU6 they would bulk up the zygomatic (upper) cheek. By bringing in AU7 they would start to narrow the eyes seductively. And so on.
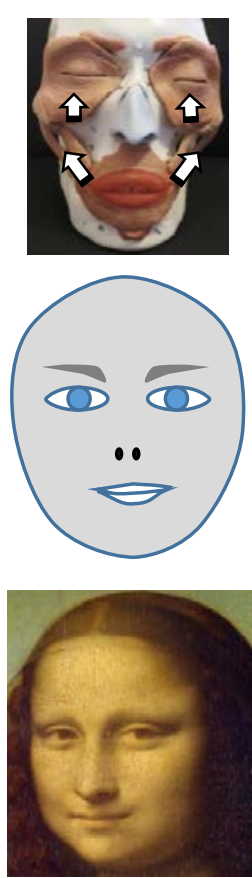

(a)

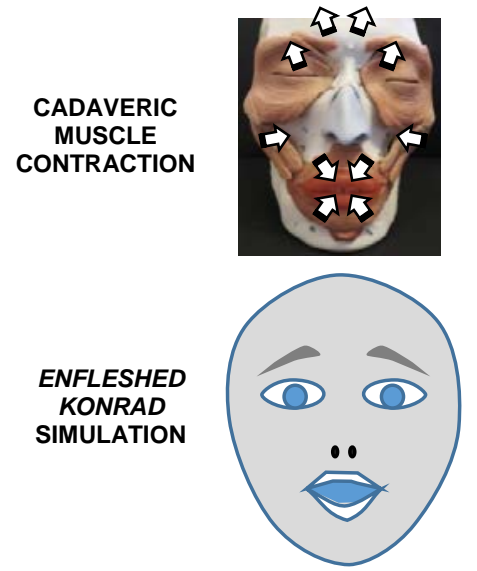

ARTIST'S IMAGINING

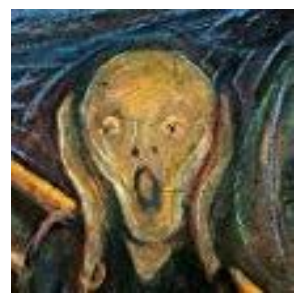

(b)
Figure 3: The muscle contractions involved in producing (a) the Mona Lisa's vaguely asymmetrical smile, and (b) The Screamer's gasp of sublime horror 


\subsection{Session B - multi-stimulus trials}

The second part of the workshop allows delegates to experiment with multi-stimulus sequences, where the facial expression generated by the first stimulus is not allowed to die away before a second, perhaps contradictory, stimulus is submitted. Then perhaps a third, and so on. In this way, novel combinations such as <STRONG FEAR WITH A LITTLE ANGRY DISGUST> can be generated. For example, in Edvard Munch's (1893) expressionist work "The Scream", the artist was attempting to set down on canvas his artistic imagining of (in his own words) "an infinite scream passing through nature" when viewing a particularly blood-red sunset over a Norwegian fjord. For our present purposes, we take that imagining as an example of a sublime emotion, namely one in which the initial response is so extreme that it momentarily overcomes the higher intellect's powers to rationalise it. The surprise component of the final horrified gasp (remember that Munch was responding to Nature's scream, and not screaming himself), is shown in Figure 3b.

\subsection{Session B - artistic intention}

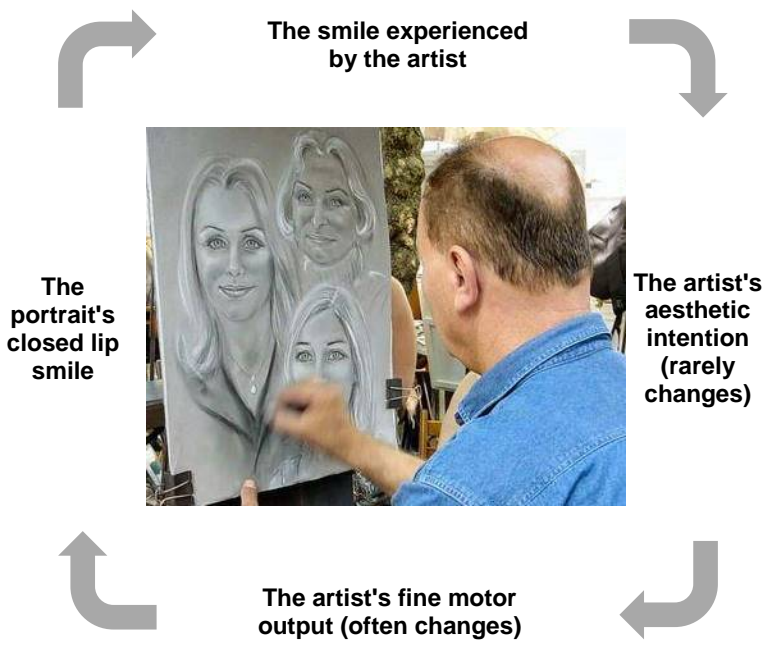

Figure 4: The Do-Review Loop in facial portraiture, seen as a cyclical process of a thousand re-touches.

How then might the Konrad software help explain the strange fact that there are only 50 verbal labels for the $\mathbf{3 0 0 0}$ possible facial expressions? Can it, for example, generate the 2950 human facial emojis, which have no single word name - expressions such as <ON THE DISGUSTED SIDE OF SAD>? And what, if anything, can mere software tell us about aesthetic philosophy? In search of insights, the workshop concludes with a theoretical discussion. When artists paint facial expressions, they do so in a recursive do-see-improve loop, only approving the end-product when it "says" back to them what they set out to "say" to their eventual audience. In other words, they begin with a single initial "artistic intention", but to deliver it requires a number - perhaps, even, a great number - of looped outputs and confirmatory re-inputs. The cyclicality for producing a smile, which says what the artist wanted it to say, is shown in Figure 4, but the underlying principle is the same for all expressions.

The theoretical discussion also allows delegates to share experiences arising from their experiments with multi-stimulus inputs. Indeed, the group as a whole is encouraged to make suggestions as to appropriate single word names for faces, which do not yet have a name. Certificatory print-outs will be made available to participants recording (a) the input parameters passed to the mainframe, (b) the output codes received back from the mainframe, (c) the cadaveric and flesh-rendered facial expressions obtained on the end-user device, and (d) the name proposed for that expression, if novel. The relevance to aesthetic theory lies in the observation that the output codes mentioned in (b) need be no more complex than the alphanumeric string "AU12L2R1", yet can elicit something as enduringly pleasing as the Mona Lisa Smile. Implications for the development of genuinely intelligent screen avatars are also discussed.

\section{REFERENCES}

Carmel Barnea Brezner Jonas (2020) http://www.carmelbbj.com/ (retrieved 3 May 2020).

Desperately Seeking (2020)

http://desperatelyseeking.online/ (retrieved 3 May 2020).

Ekman, P., Friesen, W. V., and Hager, J. C. (2002) Facial Action Coding System. UT Research Nexus, Salt Lake City.

Gonzalez Diaz, C., Perry, P., and Fiebrink, R. (2019) Interactive Machine Learning for More Expressive Game Interactions. 2019 IEEE Conference on Games (CoG), London, United Kingdom, August 2019, pp. 1-2. IEEE.
Gabriel
$\mathrm{S}$.
Moses
http://www.gabsmoses.com(retrieved 3 May 2020).

Gillies, M. (2019) Understanding the Role of Interactive Machine Learning in Movement Interaction Design. ACM Transactions on Computer-Human Interaction. 26, 1, pp. 1-34.

Höök, K. (2018) Designing with the Body Somaesthetic Interaction Design. MIT Press, Cambridge, Massachusetts.

Mahanaim134 (2019) Eyal Gruss: WanderGAN Live performance @ Trauma Night \#2. https://youtu.be/AT8fDFnXFKM (retrieved 3 May 2020). 
Mahanaim134 (2020) Eyal Gruss: Glitch Canvas. https://youtu.be/en9GHonvbOY (retrieved 3 May 2020).

Marquez Segura, E., Vidal, L. T., Rostami, A., and Waern, A. (2016) Embodied Sketching. CHI Conference on Human Factors in Computing Systems, San Jose California USA, 2016, pp. 6014-602.

Shmoogle (2020) https://shmoogle.world/ (retrieved 3 May 2020).
Smith, C. H. (2019) Carl H Smith - Exploring Double Consciousness using Holotech to Develop Hyperhumanism. https://youtu.be/MTjrJPZa5lo (retrieved 3 May 2020).

Smith, D. J. (2014). Trench Gothic: The computer visualisation of a disturbing Great War artwork. EVA2014 Conference, 9th July, BCS HQ, London.

Smith, D. J. (2016). The remarkably difficult psychology of creative visualisation. EVA2016 Conference, 14th July, BCS HQ, London. 